\title{
La lecture critique des médias : une stratégie de formation en éducation relative à l'environnement
}

Mariana Tambellini Faustino et Rosana Louro Ferreira Silva

\section{(2) OpenEdition}

Édition électronique

URL : http://journals.openedition.org/ere/6018

DOI : $10.4000 /$ ere.6018

ISSN : 2561-2271

Éditeur

Centr'ERE

\section{Référence électronique}

Mariana Tambellini Faustino et Rosana Louro Ferreira Silva, «La lecture critique des médias : une stratégie de formation en éducation relative à l'environnement », Éducation relative à l'environnement [En ligne], Volume 15 - 2 | 2020, mis en ligne le 15 novembre 2020, consulté le 25 février 2021. URL http://journals.openedition.org/ere/6018 ; DOI : https://doi.org/10.4000/ere.6018 


\title{
La lecture critique des médias : une stratégie de formation en éducation relative à l'environnement
}

\author{
Mariana Tambellini Faustino et Rosana Louro Ferreira Silva
}

1 L'éducation relative à l'environnement (ERE) au Brésil a été institutionnalisée en 1999 dans le cadre de la Politique nationale d'éducation relative à l'environnement (loi 9795/99). Cette loi indique que l'ERE doit être développée en tant que pratique éducative intégrée, continue et permanente à tous les niveaux de l'éducation formelle, ainsi que dans le contexte de l'éducation non formelle (Brasil, 1999).

2 Il y a plus d'une décennie, Guimarães (2007) avait déjà souligné que des projets et d'autres activités en ERE ont pris de plus grandes proportions et se sont propagés dans les écoles, de telle sorte que même si les contextes dans lesquels il est possible d'effectuer l'ERE se sont diversifiés et ont pris de l'ampleur, le milieu scolaire reste le principal cadre de ce type d'action éducative au Brésil (Torales, 2013).

3 Malgré le fait que l'ERE au Brésil s'est déployée jusqu'ici à travers une diversité de réalisations et de pratiques porteuses dans différents contextes d'action, nous traversons actuellement une période d'incertitudes et d'inquiétudes en raison des changements et de l'affaiblissement des politiques publiques visant la conservation de l'environnement et l'ERE. Les actions liées à la sphère politique actuellement en place au Brésil sont étroitement surveillées par de nombreuses institutions de protection de l'environnement, des gouvernements et des médias du monde entier en raison de la négligence de l'actuel gouvernement brésilien en matière d'environnement.

4 La période actuelle nous invite encore davantage à repenser la relation entre les faits et la manière dont ils sont véhiculés dans les médias. Dans le contexte brésilien, le Traité sur l'éducation relative à l'environnement pour des sociétés durables et de responsabilité mondiale (Brasil, 2005) mentionnait déjà l'utilisation des médias comme outils éducatifs pour la préservation et la conservation des ressources naturelles. L'utilisation et la production de médias dans des contextes scolaires brésiliens sont aussi prévues dans la 
Résolution $\mathrm{n}^{\circ}$ 2/2012 (Brasil, 2012), un document d'orientation pour l'enseignement secondaire.

Dans ce contexte, il est utile d'étudier comment les enseignant.e.s se préparent à la problématisation critique de contenus liés aux enjeux environnementaux, tels que la diminution de la biodiversité, la déforestation et les catastrophes socioenvironnementales, qui sont souvent véhiculées par les médias. À ce titre, Reigota (1999, p. 109) suggère que « les professionnels de l'éducation relative à l'environnement devraient s'efforcer de discuter de ce que les médias représentent et diffusent " (traduction libre), et que soit reconnue l'importance d'une formation des enseignant.e.s concernant la pratique de la lecture critique des médias afin qu'ils puissent contribuer à la formation d'une société plus consciente et plus active en ce qui concerne les enjeux socio-environnementaux.

6 C'est en ce sens que nous avons expérimenté une pratique de lecture critique des médias dans le cadre d'un atelier de formation continue des enseignant.e.s du secondaire du système d'enseignement public de l'État de São Paulo. L'objectif de cette recherche est de caractériser les discussions au sein du groupe des enseignant.e.s au cours de cette activité, en particulier au regard du courant critique de l'éducation relative à l'environnement.

\section{Cadre théorique}

7 Les approches scolaires en ERE varient énormément, allant de pratiques ponctuelles axées sur une pédagogie traditionnelle, à des projets interdisciplinaires plus holistiques et critiques. C'est en ce sens que nous accordons une grande importance au travail développé par Sauvé (2006) qui énumère les caractéristiques de quinze différents courants d'ERE et qui clarifie le large spectre de théories et pratiques associées à l'ERE.

Parmi les quinze courants, notre approche rejoint davantage celui de l'ERE critique (Sauvé, 2017, 2006 ; Carvalho, 2012 ; Guimarães, 2007 ; Silva, 2007), centré sur l'analyse des dynamiques sociales associées aux réalités et aux enjeux environnementaux (Sauvé, 2006). Selon cette auteure, l'ERE critique vise à

contribuer à la formation d'une citoyenneté consciente des liens étroits entre société et nature, une citoyenneté critique, créative et engagée, compétente et désireuse de participer aux débats publics et à la transformation des politiques et des pratiques éco-sociales (Sauvé, 2017, p. 5)

De plus, Sauvé et Orellana (2008) soutiennent que l'enseignement et l'apprentissage de la pensée critique visent l'autonomie, associée à une autodéfense intellectuelle et à une liberté de pensée. Par ailleurs, la pédagogie critique est orientée vers des valeurs sociales telles que la justice, l'équité, la démocratie et l'émancipation collective.

Dans cette perspective, Carvalho (2006) considère la dimension politique comme la caractéristique centrale d'un processus éducatif en ERE, soulignant également l'importance d'inclure dans les pratiques les trois éléments suivants: les " connaissances », les «valeurs esthétiques et éthiques» et la "participation». La Figure 1 présente ces dimensions de la pratique éducative selon Carvalho (2006). 

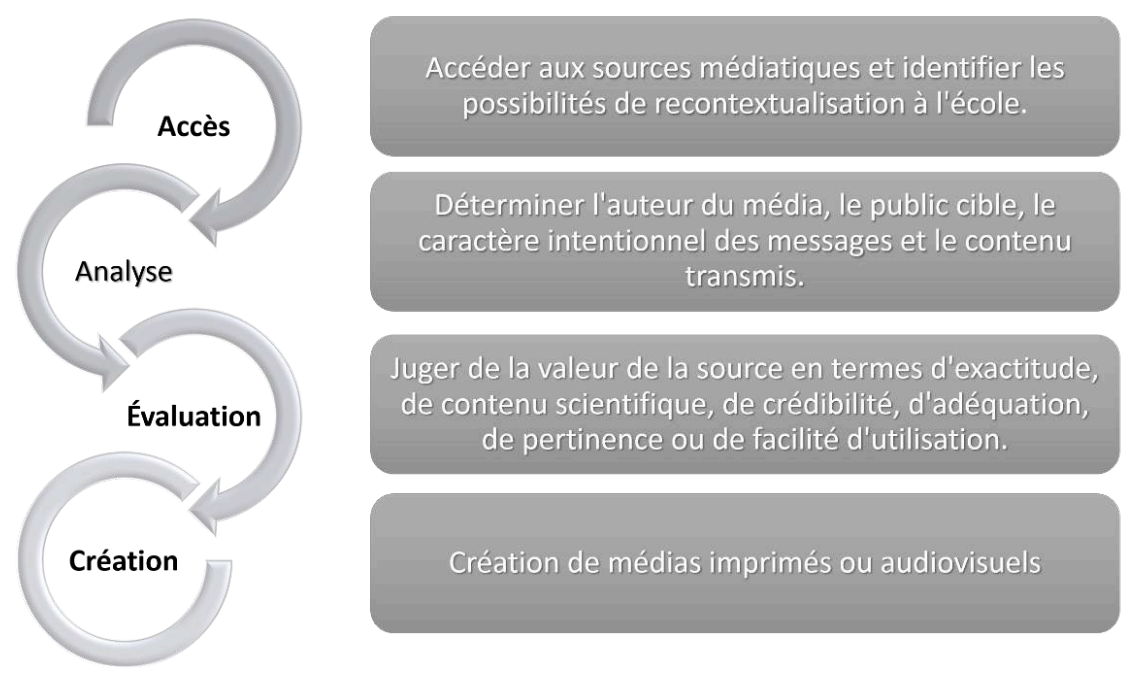

Figure 1 : Catégories d'utilisation des médias. Source : adapté par Klosterman, Sadler et Brown (2012) [traduction libre].

11 Également, les travaux de Paulo Freire, en renforçant la critique de toutes les structures économiques et sociales actuelles qu'il importe de transformer, offrent une base théorique importante pour l'ERE critique au Brésil. Dans son ouvrage intitulé Pédagogie de l'autonomie (Freire, 2001), bien qu'il n'utilise pas directement le terme "éducation relative à l'environnement ", l'auteur décrit les relations extrêmement importantes entre la politique, l'action et l'environnement, soit en critiquant et en dénonçant les inégalités, soit en conscientisant les enseignant.e.s aux réalités socioenvironnementales dans lesquelles ils travaillent.

En marge des réels efforts déployés par les éducateurs dans les contextes formels et non formels de l'éducation pour sensibiliser et encourager les actions et les attitudes appropriées face aux enjeux socio-environnementaux actuels, il importe de souligner le rôle joué par les médias quant aux informations véhiculées à propos de l'environnement. À cet effet, Carvalho (2012) montre qu'il est assez courant pour la population d'avoir des représentations naturalistes et naïves de l'environnement en raison des représentations imaginaires créées par les productions médiatiques.

Cette recherche est donc également basée sur les fondements théoriques de l'éducation aux médias et à l'information (EMI). En 2012, l'UNESCO a lancé le programme de formation pour les enseignant.e.s sur l'EMI dans lequel on retrouve l'extrait suivant :

L'éducation aux médias et à l'information regroupe des connaissances essentielles sur (a) les fonctions des médias et des autres diffuseurs d'information dans les sociétés démocratiques, (b) les conditions dans lesquelles les médias d'information et les diffuseurs d'information peuvent effectivement remplir ces fonctions, et (c) la façon d'apprécier comment ces fonctions sont remplies en évaluant les contenus et les services qu'ils offrent (UNESCO, 2012, p. 18).

Toujours dans la perspective de l'EMI, Klosterman, Sadler et Brown (2012) identifient quatre modes d'utilisation des médias dans les contextes scolaires: l'accès, l'analyse, l'évaluation et la création (Figure 2). 


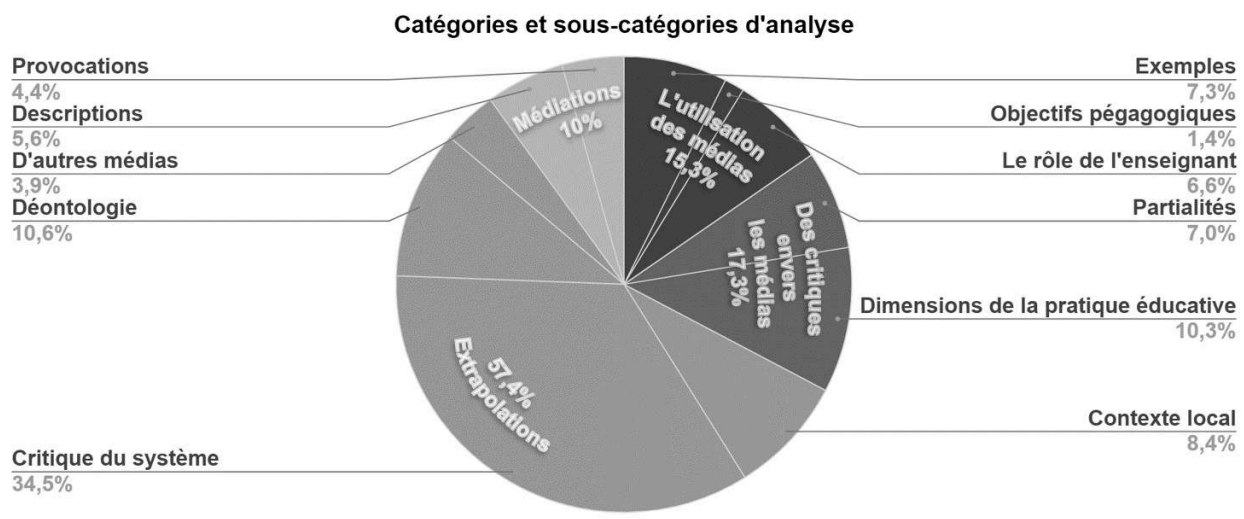

Figure 2 : Catégories et sous-catégories d'analyse de la discussion avec les enseignant.e.s. Pourcentage de la couverture. Source : données de la recherche.

La pratique de la lecture critique des médias correspond à l'ensemble des actions permettant d'y accéder, de les analyser et de les évaluer (qu'il s'agisse de reportages télévisés, d'extraits de journal, de publicités, de textes de blogue, de vidéos documentaires, etc.). Lorsqu'un.e enseignant.e propose une lecture critique de ce qui a été véhiculé par les médias, les élèves sont invités une faire une nouvelle lecture du monde, en plus de mieux comprendre la puissance des médias et le rôle qu'ils occupent dans l'espace public (Caldas, 2006).

Selon Arroio (2017), les pratiques d'EMI peuvent encourager la pensée critique et la liberté d'expression, en valorisant l'équité entre les genres, la justice sociale et la participation civique dans une société démocratique. Encore une fois, il est possible d'y percevoir l'héritage de la pensée de Paulo Freire. Promouvoir l'exercice de la lecture critique des médias ou inviter les élèves et les enseignant.e.s à développer des productions médiatiques s'inscrit dans une vision libératrice, critique, participative et émancipatrice de l'éducation.

Il n'est donc pas surprenant que Paulo Freire lui-même ait déjà montré la pertinence de démystifier ce qui est véhiculé par les médias, en particulier la télévision, dans les contextes scolaires :

Débattre de ce qui est dit, de ce qui est montré et de comment cela est montré à la télévision me semble être quelque chose de plus en plus important [...]. En tant qu'éducateurs progressistes, non seulement nous ne pouvons pas ignorer la télévision, mais nous devons l'utiliser et, surtout, en discuter (Freire, 2001, p. 157, traduction libre).

Signalons que diverses recherches ont été menées sur la question de l'EMI par les membres de notre Groupe de recherche en éducation relative à l'environnement et en formation des éducateurs (Grupo de Pesquisa em Educação Ambiental e Formação de Educadores - GPEAFE) de l'Université de São Paulo au Brésil.

19 L'une de nos études montre la difficulté des enseignant.e.s en formation initiale à concevoir des processus d'appropriation critique et significative des médias en classe (Faustino et Silva, 2013). Le travail de recherche de de Silva (2010), qui a développé une analyse des images de couverture de magazines liées à des questions socioenvironnementales en contexte de formation continue des enseignant.e.s, a montré la 
nécessité d'améliorer la formation en ce qui a trait à l'analyse critique et à l'utilisation des médias dans des contextes scolaires.

Une autre étude portant sur la formation initiale des enseignant.e.s (Silva et Abreu, 2015) a montré que pour transformer un média en ressource didactique, il faut envisager diverses stratégies d'enseignement - impliquant des aspects de médiation, de recontextualisation et d'interactions discursives.

Thinen et Silva (2014) ont identifié une pluralité d'approches dans les films d'un festival audiovisuel environnemental brésilien, invitant à identifier et à discuter des concepts scientifiques et des valeurs véhiculées dans les productions. Finalement, dans le cadre du travail de recherche de Roberto (2019), des élèves du secondaire ont analysé une production média et les diverses interprétations de celle-ci ont été mises en évidence.

La recherche que nous présentons ici s'inscrit dans la foulée de ces diverses études.

\section{Méthodologie}

Comme nous l'avons déjà mentionné, notre recherche consiste en l'analyse d'une pratique de lecture critique d'un documentaire réalisée au cours d'un atelier de formation continue des enseignant.e.s du secondaire. Cet atelier a eu lieu en octobre 2016 dans le cadre d'un partenariat entre le GPEAFE et le Bureau régional d'éducation de l'État de São Paulo (Diretoria de Ensino Regional do Estado de São Paulo - DER). Le groupe de participant.e.s était composé de 16 enseignant.e.s de différents domaines disciplinaires.

Le thème de l'atelier était l'utilisation des médias sur des questions environnementales en contexte scolaire. Au cours de cet atelier, nous avons élaboré une activité de lecture critique d'un média télévisuel ${ }^{1}$ portant sur la biodiversité. Parmi les nombreux thèmes qui peuvent être abordés en ERE, nous croyons, tout comme Kawasaki et Oliveira (2003), que celui de la biodiversité dépasse le cadre des sciences biologiques et peut faire l'objet d'une approche interdisciplinaire. Également, traiter de la biodiversité invite à tenir compte des aspects idéologiques, scientifiques, économiques et politiques qui y sont liés (Gómez et Bernat, 2010).

Il est important de noter que la production médiatique sur laquelle nous nous sommes concentré.es n'a pas été produite à des fins éducatives : il s'agit d'un reportage présenté dans le cadre d'une émission télévisée destinée aux familles, qui associe journalisme, sports, humour, science et curiosités.

Les enseignant.e.s ont visionné le reportage pour ensuite en discuter après la projection. La discussion était animée par la chercheure, l'une des auteures de cet article, qui a également partagé avec les enseignant.e.s une grille d'analyse. Pour la confection de cette grille d'analyse, nous nous sommes approprié certaines dimensions de la pratique éducative exposées par Carvalho (2006) et les catégories de l'EMI de Klosterman, Sadler et Brown (2012).

Cette recherche-intervention (Van Der Maren, 1996) est axée sur une approche méthodologique qualitative permettant l'analyse du discours et des actions des participants à la recherche (Bogdan et Biklen, 2010). L'enregistrement audio de la discussion avec le groupe enseignant a donc été transcrit en vue d'en faire une analyse de contenu (Bardin, 1977). Mentionnons que la recherche a été approuvée par un 
Comité d'éthique ${ }^{2}$ et que les enseignant.e.s ont accepté librement de participer à la recherche.

Il est important de signaler que pendant la période de planification de la formation, c'est-à-dire avant de réaliser l'activité de lecture critique du reportage, nous imaginions que le point central de la discussion du groupe enseignant serait celui des trois composantes de la pratique éducative identifiées par Carvalho (2006): connaissances, valeurs éthiques et esthétiques, et participation. Cependant, lors de l'analyse de la transcription des échanges après l'activité, nous avons réalisé que la discussion avait été beaucoup plus diversifiée, de sorte que nous avons décidé de construire une catégorisation a posteriori afin de mieux caractériser les propos des participant.e.s.

D'abord, la transcription a été divisée en unités d'analyse, soit en extraits comprenant les énoncés d'un.e seul.e enseignant.e ou un bref moment d'interaction. Pour faciliter la catégorisation a posteriori des unités d'analyse, nous avons utilisé le logiciel NVIVO ${ }^{3}$. La catégorisation a donc émergé des données de recherche. L'utilisation du logiciel a été importante pour l'organisation des données et pour la détermination du pourcentage de couverture de chaque catégorie et sous-catégorie.

À partir de l'analyse de la transcription, nous avons identifié 4 catégories, chacune comportant un certain nombre de sous-catégories: utilisation des médias; critiques envers le reportage; extrapolations; interventions de l'animatrice. Afin d'éliminer d'éventuels biais dans l'analyse, puisque l'une des chercheures a participé en tant qu'animatrice de la discussion, l'ensemble du travail de catégorisation a été mis à la disposition de tous les membres du GPEAFE, qui ont validé les analyses présentées ici.

\section{Résultats et discussion}

31 Les catégories, les sous-catégories et leurs pourcentages de couverture de la discussion au sein du groupe enseignant après le visionnement du reportage sur la biodiversité sont présentés à la Figure 3 : 


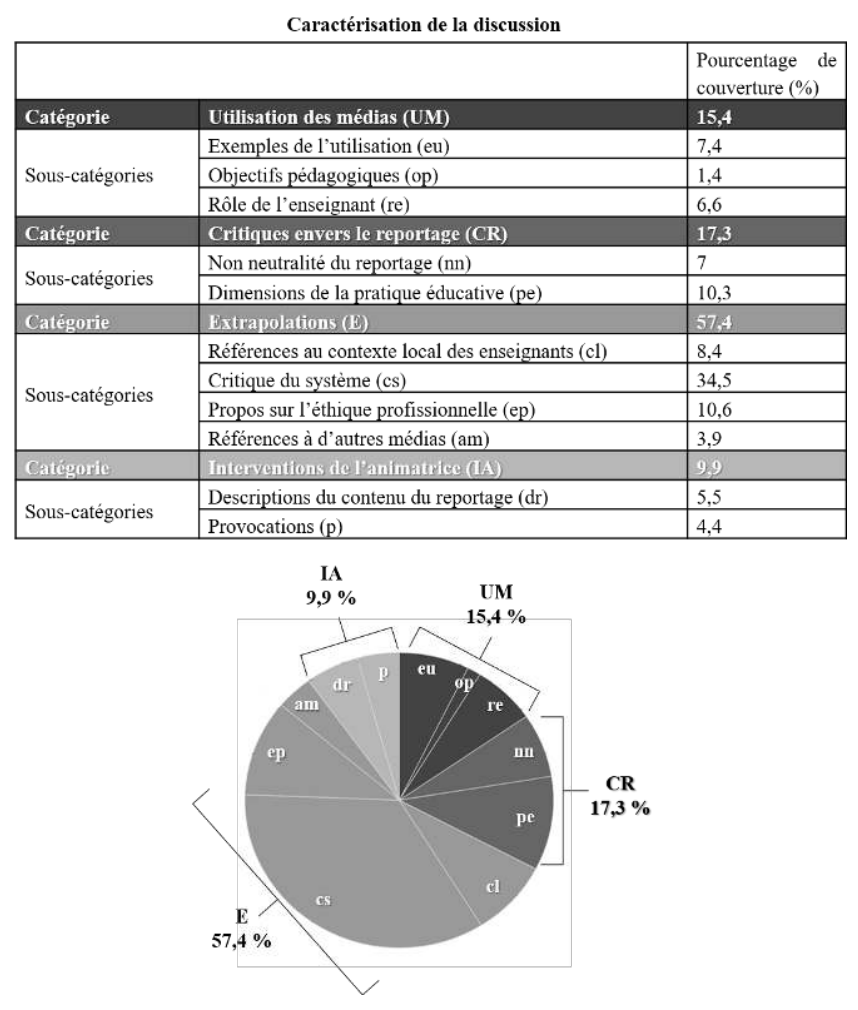

Figure 3 : Catégories, sous-catégories et leurs pourcentages de couverture respectifs

\section{Utilisation des médias}

Dans la catégorie "Utilisation des médias", qui correspond à un pourcentage de couverture de 15,3\% de l'ensemble des éléments de la discussion, toutes les unités d'analyse qui font référence à des objectifs pédagogiques d'utilisation des médias en général et aussi spécifiquement de ce reportage, ont été prises en considération. Nous y avons également inclus des unités d'analyse indiquant des exemples de la façon dont les enseignant.e.s utiliseraient le reportage à l'école et le rôle que ceux-ci devraient jouer dans une telle situation pédagogique.

Le premier objectif pédagogique mentionné par le groupe enseignant est l'utilisation des médias comme ressource pour introduire un sujet ou un thème en classe, une pratique récurrente dans les contextes scolaires. Faustino et Silva (2013) soulignent que les futurs enseignant.e.s dans le contexte de stages à l'école utilisent les médias pour faciliter le rapprochement entre les contenus enseignés et la réalité des étudiant.e.s. Il est important de signaler qu'au départ, les médias ne sont pas conçus pour être présentés dans un contexte éducatif. À ce sujet, Marandino, Selles et Ferreira (2009) montrent l'importance $\mathrm{du}$ rôle de de l'enseignant.e pour s'assurer de la recontextualisation des médias à l'école - c'est-à-dire pour en relocaliser le contenu dans le contexte éducatif -, ainsi que pour établir les visées, le sens et les spécificités de leur utilisation.

Le groupe enseignant a également mentionné un deuxième objectif, celui de discuter en classe des erreurs possibles rapportées par les médias. Xavier et Kerr (2004) préviennent que les médias, en transmettant des contenus liés à l'environnement, 
peuvent cristalliser des erreurs conceptuelles auprès de la population. En ce sens, tout travail pédagogique qui vise une réflexion critique sur les médias s'avère pertinent.

Lorsque nous associons ces objectifs aux catégories de l'EMI de Klosterman, Sadler et Brown (2012), il est possible de constater que le premier objectif mentionné, qui est associé à l'action de contextualiser et d'illustrer certains contenus, est davantage lié à la catégorie de base, l'accès. Le deuxième objectif offre un plus grand potentiel pour réfléchir au processus de médiatisation et s'approche en ce sens d'une évaluation des médias.

Bien que l'accès aux médias permet déjà une utilisation qui, en soi, est pertinente et valorisée dans les contextes scolaires pour favoriser les processus d'enseignement et d'apprentissage, nous soutenons que les approches plus complexes, telles que les évaluations et les créations médiatiques, peuvent offrir de meilleures opportunités pour développer l'autonomie et contribuer à la formation de citoyens conscients et critiques de ce qui est propagé par les médias.

Quant aux exemples d'utilisation du reportage mentionnés lors des échanges, un enseignant a suggéré une discussion critique sur la valorisation anthropique et économique de la biodiversité et la possibilité d'établir un parallèle assez intéressant entre cette question et l'esclavage. L'argument de l'enseignant est basé sur le fait que les esclaves étaient également considérés comme une ressource dont la présence et le travail étaient uniquement destinés au plaisir et au bénéfice des hommes de la classe dirigeante. Dans ce cas, nous voyons clairement que l'ERE est envisagée dans une perspective plus holistique et multidisciplinaire.

Toujours dans la catégorie "Utilisation des médias", nous avons inclus des unités d'analyse qui font référence au rôle de l'enseignant.e. L'importance de savoir sélectionner un média pour son utilisation à l'école a été soulignée par le groupe enseignant.

Sur cette question, Paulo Freire (2001, p. 157) affirme ceci :

Je ne crains pas de paraître naïf en insistant sur le fait qu'il n'est même pas possible de penser à la télévision sans tenir compte de la question de la conscience critique. Penser à la télévision ou aux médias en général nous pose le problème de la communication, dont la neutralité est impossible.

Cet auteur insiste sur le fait que capter et confronter les idéologies qui se trouvent derrière les discours télévisés n'est pas une tâche facile parce qu'elle exige d'être alerte en tout temps. Il dénonce également les pratiques naïves concernant ce moyen de communication (Freire, 2001).

41 De cette façon, nous soulignons l'importance du travail de l'enseignant.e qui doit pouvoir être attentif aux discours télévisés (ou provenant d'autres médias), et être capable d'en faire l'analyse et l'évaluation avant de planifier son utilisation pédagogique. Ce faisant, il augmente et diversifie les possibilités d'apprentissage et de prise de conscience critique des contenus véhiculés par les médias, tant pour lui-même que pour ses élèves.

Pour terminer cette sous-section, mentionnons également que les enseignant.e.s ont traité de leur propre rôle dans la construction de l'autonomie des élèves. Rappelons à cet effet le passage suivant du livre La pédagogie de l'autonomie :

L'autonomie en tant qu'une maturité de l'être envers soi-même est un processus, un devenir. Elle ne se produit pas à une date prévue. C'est en ce sens qu'une pédagogie de l'autonomie doit se concentrer sur des expériences qui stimulent la prise de 
décision et la responsabilité, c'est-à-dire sur des expériences qui respectent la liberté (Freire, 2001, p. 121, traduction libre).

\section{Critiques envers le reportage}

43 Dans la deuxième catégorie, "Critiques envers le reportage", qui comprend un pourcentage de couverture de 17,3\% de l'ensemble de la discussion, nous avons inclus les unités d'analyse qui concernent les critiques formulées par le groupe enseignant en lien avec les biais du reportage. Nous avons également inclus les unités d'analyse qui traitent de la critique de la pratique éducative (Carvalho, 2006). Il est important de rappeler qu'au cours de la discussion, les enseignant.e.s avaient en main une grille d'analyse dont les questions ont stimulé l'interaction entre eux.

Concernant les biais médiatiques et la non-neutralité du reportage, le groupe enseignant a discuté d'un extrait montrant les différents degrés de déforestation de l'Amazonie. Le territoire forestier le plus préservé était entretenu par une industrie minière privée et celui le moins préservé était un territoire géré par le gouvernement.

Les enseignant.e.s ont considéré que l'extrait était biaisé, observant que le reportage développait un discours implicite en faveur de la privatisation des zones vertes, puisqu'il y est mentionné que le territoire forestier sous la gouverne du secteur privé est mieux entretenu. Le groupe enseignant a jugé cette information douteuse parce qu'elle était déconnectée du reste du reportage et qu'aucune autre donnée n'a été fournie pour confirmer cette affirmation.

L'analyse de la discussion entre les participant.e.s a ainsi permis de constater que les enseignant.e.s montrent des signes de prise de conscience concernant la possible partialité des médias. Pour Henning, Garré et Henning (2010, p. 246),

en ordonnant et en constituant la réalité, les médias fabriquent des modes de vie.

Ils sélectionnent ce qui doit être dit et indiquent comment cela doit être dit. Ainsi,

le média met en œuvre une opération de pouvoir qui affecte beaucoup de gens (...).

(Traduction libre).

47 Le fait que le reportage ait été diffusé sur le plus grand réseau ouvert de la télévision brésilienne illustre bien cette situation. Le groupe enseignant a relevé la puissance de l'influence de ce média. Santos (2006) souligne à cet effet que la façon dont les médias sélectionnent, révisent et rapportent certains événements fait toute la différence dans la compréhension par le public de ce qui est véhiculé.

En ce qui concerne la critique portant sur les dimensions de la pratique éducative, l'animatrice a demandé aux enseignant.e.s s'ils et elles avaient pu repérer des éléments qui pourraient être associés aux « connaissances, valeurs éthiques et esthétiques et à la participation » (Carvalho, 2006).

Le groupe enseignant a estimé que le reportage n'était pas très riche en " connaissances ». Le seul passage relevé est celui où un journaliste demande à un enseignant ce qu'est la biodiversité.

Un participant à la formation a critiqué la perspective adoptée par le reportage concernant la relation entre l'être humain et l'environnement, lui reprochant de ne pas montrer l'être humain comme faisant partie intégrante de l'environnement. Selon la typologie établie par Silva (2007), nous avons observé qu'une telle approche du reportage s'éloigne d'une perspective d'ERE critique, car elle ne présente pas les 
interactions complexes entre l'être humain et l'environnement, qui vont bien au-delà du simple réductionnisme biologique.

Quant à la dimension "valeurs éthiques et esthétiques », le groupe enseignant a relevé l'importance accordée par les médias à la marchandisation de la biodiversité. Plusieurs exemples ont été signalés, comme le fait que l'acajou est un arbre très apprécié dans le secteur de la construction de meubles, ou encore que le venin de certains serpents est essentiel à la recherche et au développement de nouveaux médicaments. Une discussion a donc été engagée sur le caractère anthropocentrique et économique de la valeur de la biodiversité telle que présentée par le reportage. Enfin, les enseignant.e.s ont déclaré n'avoir trouvé dans le reportage aucun élément lié à la dimension «participation ».

\section{Extrapolations}

Dans la catégorie «Extrapolations », qui regroupe 57,4 \% des éléments de la discussion, nous avons pris en compte les unités d'analyse qui présentent des sujets ou des contenus apparus dans la discussion avec les enseignant.e.s, qui n'étaient manifestement pas abordés dans le reportage et qui ne correspondaient pas à des commentaires critiques à propos de celui-ci. À titre d'exemple, le groupe enseignant a discuté du contexte de travail et de l'éthique professionnelle, a critiqué le système économique actuel et a suggéré d'autres productions diffusées dans les médias qui pourraient être utilisées en classe.

La première sous-catégorie regroupe les unités d'analyse qui se rapportent au contexte local des enseignant.e.s, y compris l'indication de certaines des difficultés rencontrées par ceux-ci lorsqu'ils abordent les enjeux environnementaux dans leur école. Le groupe a discuté du fait que les questions liées à l'Amazonie peuvent être lointaines pour un.e étudiant.e qui vit en banlieue à São Paulo. Dans les régions où travaillent ces enseignant.e.s, la croissance urbaine a été mal planifiée, de sorte que le contact avec les espaces verts est très rare dans la vie de leurs élèves. .

Paulo Freire (2001) explique que les conditions dans lesquelles vivent les apprenants influencent la compréhension de leur propre monde ainsi que leur capacité d'apprendre et de relever des défis. À ce sujet, certains enseignants ressentent de l'impuissance face au défi d'aborder des questions environnementales qui échappent à la réalité des élèves. L'un des participants manifeste même un certain défaitisme quant à la possibilité d'intéresser les élèves à de telles questions.

Toutefois, Freire (2001) apporte une réflexion qui peut susciter l'espoir : il témoigne de l'importance que peuvent avoir les enseignant.e.s qui travaillent dans une perspective politico-pédagogique capable de former des citoyens critiques, conscients «d'être conditionnés, mais en même temps capables de surmonter leur propre conditionnement» (p. 146, traduction libre). Toutefois, les enseignant.e.s ne devraient pas penser naïvement qu'à partir « du cours qu'ils coordonnent ou du séminaire qu'ils dirigent, ils peuvent transformer le pays ; mais ils peuvent démontrer qu'il est possible de changer » (p. 126, traduction libre).

Un aspect des échanges entre les participant.e.s a particulièrement retenu notre attention : un tiers de l'ensemble de la discussion était centré autour de thématiques qui ont été classées dans la sous-catégorie "Critiques du système ». On retrouve dans cette sous-catégorie, des unités d'analyse relevant d'une critique du système 
économique dominant ainsi que des questions connexes, telles que la consommation, la durabilité des produits et le mode de vie.

Le groupe enseignant a soulevé des problématiques liées aux mécanismes de consommation excessive et d'obsolescence programmée. À cet effet, Carvalho (2012) identifie comme alternative à cette société capitaliste de consommation, le rejet de la rationalité instrumentale, de l'individualisme et de la logique coût-bénéfice purement économique, rejet qui caractérise une société écologique.

La critique nécessaire de cette consommation excessive est également mise en évidence par Zacarias (2009, p. 135, traduction libre) :

Les modes de production et de consommation créent d'importantes répercussions sociales et environnementales. Dans ce contexte, on peut affirmer que la lutte pour une société plus démocratique implique, entre autres, une critique du consumérisme et surtout, la dénonciation de l'iniquité dans la répartition de la consommation sur la planète.

À cet effet, un enseignant a mentionné qu'il suggère aux élèves d'adopter un mode de consommation consciente et durable. Certes, une telle perspective exige un travail approfondi et continu sur le sujet, afin que les étudiant.e.s développent entre autres un sens critique leur permettant ne pas tomber dans le piège d'un «verdissement » de surface.

60 À ce sujet, Fontanelle (2013) souligne que les médias utilisent souvent la culpabilité ressentie par la population à l'égard des enjeux socio-environnementaux, qui est habituellement le résultat des discours catastrophistes véhiculés par les médias euxmêmes, afin d'encourager une économie et une consommation qu'ils prétendent responsables. L'auteure affirme également que le consommateur, lorsqu'il achète des produits d'entreprises qui se disent respectueuses de l'environnement, peut avoir droit à une sorte de rédemption qui le fait se sentir soulagé et exempté de toute autre responsabilité dans la lutte contre les enjeux socio-environnementaux.

61 Le groupe enseignant a discuté du fait que les entreprises abordent le nouveau paradigme de la responsabilité environnementale avec de grands intérêts économiques, car la bonne image médiatique qui en résulte est assortie d'incitatifs fiscaux autorisés par le gouvernement brésilien. Une telle « vitrine verte » est également présentée dans les médias du secteur économique en tant que nouvelle source d'affaires capable d'accroître la compétitivité du Brésil et de permettre également de bons retours sur investissement (Santos, 2006).

62 La troisième sous-catégorie d'énoncés présente une réflexion sur la responsabilité éthique de l'enseignant.e. Selon les participant.e.s, il est inopportun d'enseigner des comportements considérés politiquement corrects aux étudiants si le professeur luimême ne s'engage pas à les mettre en pratique.

Paulo Freire (2001, p. 17) est d'avis que la meilleure façon pour un.e enseignant.e de promouvoir une certaine éthique "est de la vivre dans sa propre pratique et d'en témoigner d'une manière vivante » (traduction libre), c'est-à-dire de montrer l'exemple afin d'exiger la même chose des étudiant.e.s. Par ailleurs, les auteurs Sauvé et Orellana (2008) confirment qu'il n'y a pas de développement d'une compétence critique sans développement d'une compétence éthique.

Les unités d'analyse classées dans la dernière sous-catégorie fournissent des exemples d'autres productions diffusées dans les médias que les enseignant.e.s peuvent utiliser 
en classe pour aborder les questions liées à l'environnement. Les films d'animation Wall-e et Rango et la série Les Simpson ont été mentionnés

\section{Interventions de l'animatrice}

Dans la catégorie "Interventions de l'animatrice », qui représente $10 \%$ de l'ensemble de la transcription de la discussion, nous avons considéré les unités d'analyse qui indiquent la présence d'une animation plus active par la chercheuse, que ce soit en demandant de décrire certains aspects du reportage ou en posant des questions visant à confronter ou à orienter les propos.

Dans un premier temps, l'animatrice, cherchant à reprendre certaines scènes présentées dans le reportage, a posé des questions qui ont généré exclusivement des éléments de description du contenu du documentaire sans que les enseignants ne formulent de critiques ou de commentaires.

L'animation est ensuite devenue plus directive pour susciter davantage de réflexions chez les enseignant.e.s, surtout dans les moments de l'activité où ceux-ci ont discuté de la présence ou de l'absence de dimensions éducatives dans le reportage. Comme nous l'avons mentionné précédemment, lors de la planification de l'activité, nous avions envisagé ce moment de discussion comme l'un des plus importants; c'est pourquoi l'animatrice s'est montrée plus présente et directive dans cette partie de l'échange entre les participant.e.s.

Il convient de rappeler que l'animatrice apporte également avec elle ses convictions, ses expériences personnelles et les modèles qu'elle a intégrés tout au long de sa formation professionnelle (Altarugio, 2017). Malgré ces biais possibles, nous considérons raisonnable le pourcentage de couverture de cette sous-catégorie $(4,4 \%)$, puisque cette activité prévoyait et nécessitait une animation.

\section{Conclusion}

Dans cet article, nous avons présenté la caractérisation d'une discussion entre des enseignant.e.s en formation continue dans une pratique de lecture critique des médias. Cette recherche a permis de combiner des références du domaine de l'ERE critique et de l'EMI afin de favoriser une formation axée sur les fondements d'une éducation libertaire, émancipatrice, engagée, consciente et autonome (Freire, 2001).

Nous avons donc montré les résultats d'une pratique de lecture critique d'un reportage télévisé sur la biodiversité, ayant bénéficié de la médiation d'une animatrice. Une grille d'analyse a permis d'en faire ressortir les caractéristiques. Nos résultats montrent qu'un atelier de formation centré sur une telle production médiatique peut créer des moments dans lesquels les participants mobilisent leurs connaissances, expériences et histoires de vie pour en analyser le contenu et le réinterpréter à leur propre manière. On observe que plus de $50 \%$ de la discussion a porté sur des questions qui n'étaient pas abordées par le reportage.

71 Le groupe enseignant a perçu et indiqué des éléments manifestant la non-neutralité du reportage, signe d'une prise de conscience des biais médiatiques. En référence au courant de l'ERE critique, il est intéressant de constater que les participant.e.s ont utilisé une longue période de la discussion pour échanger sur des sujets tels que la 
politique et la crise du système économique. C'est également au cours de cette période de discussion que les enseignant.e.s ont été plus encouragé.e.s à parler librement de leurs pratiques personnelles et à échanger leurs expériences avec leurs collègues.

Cependant, nous avons remarqué que d'autres aspects du reportage, comme le recours à une image déformée de la science, n'ont pas déclenché de discussion entre les enseignant.e.s. Par ailleurs, il nous apparaît important d'inclure dans de futures formations des éléments de contextualisation spatiale et temporelle rejoignant plus spécifiquement le groupe de participants, comme des réalités ou problèmes liés à la réalité locale et aux événements récents.

Nous sommes d'accord avec Silva (2010) lorsqu'elle considère qu'il est extrêmement important de former les enseignant.e.s à la lecture critique des médias, les invitant entre autres à remettre en question le processus de production et de diffusion des questions relatives à l'environnement par les médias. En outre, nous observons que l'activité de lecture critique d'une production médiatique peut inviter les enseignants à repenser leurs propres pratiques concernant l'utilisation des médias à l'école.

Finalement, il apparaît certes nécessaire d'élargir cette étude à d'autres contextes afin de mieux comprendre la façon dont la pratique de la lecture critique des médias peut contribuer au développement d'une écocitoyenneté chez les enseignant.e.s et par conséquent, chez leurs élèves, surtout si nous considérons d'une part, l'inquiétante crise environnementale actuelle et d'autre part, la grande quantité de nouvelles fausses ou biaisées à laquelle nous sommes constamment exposés.

\section{BIBLIOGRAPHIE}

Altarugio, M. H. (2017). Da prática do sujeito ao sujeito da prática : uma trajetoria para a busca de um sentido próprio acerca da reflexão docente. Dans Shigunov Neto, A. Et Fortunato, I. (Dir.). 20 anos sem Donald Schön : o que aconteceu com o professor reflexivo ? São Paulo : Edições Hipótese.

Arroio, A. (2017). Is media literacy an urgent issue in education for all ? Problems of education in the 21th century, vol. 76, $\mathrm{n}^{\circ} 5,416-418$.

Bardin, L. (1977). Análise de conteúdo. Lisboa : Edições 70.

Brasil. (2012). MEC. Resolução № 2 de 30 de janeiro de 2012. Diário Oficial da união, 24/01/2012.

Brasil. (2005). MEC/MMA. Programa Nacional de Educação Ambiental. Brasília : MEC/MMA. $3^{\text {e }}$ édition.

Brasil. (1999). Lei oํ 9795, de 27 de abril de 1999. Diário Oficial da União, 28/04/1999.

Bogdan, R. C. et Biklen, S. K. (2010). Investigação qualitativa em educação. Porto : Porto Editora.

Caldas, G. (2006). Mídia, Escola e Leitura crítica do mundo. Educ. Soc. vol. 27, nº 94, 117-130.

Carvalho, I. C. de M. (2012). Educação Ambiental a formação do sujeito ecológico. São Paulo : Cortez, $6^{\mathrm{e}}$ édition. 
Carvalho, L. M. A temática ambiental e o processo educativo : dimensões e abordagens. Dans Cinquetti, H. C. S., Logarezzi, A. (Dir.). Consumo e resíduo : fundamentos para o trabalho educativo. São Carlos : EdUFSCar.

Faustino, M. T. et Silva, R. L. F. (2013). Mídia e Ensino de Biologia : Aspectos de alfabetização científica nos planejamentos de aulas construídos por licenciandos. Enseñanza de las ciências, v. extra, 3488-3493.

Freire, P. (2001) Pedagogia da autonomia - saberes necessários à prática educativa. São Paulo : Paz e Terra. $17^{\mathrm{e}}$ édition.

Fontanelle, I. A. (2013). A ressignificação da crise ambiental pela mídia de negócios : responsabilidade empresarial e redenção pelo consumo. Galaxia Online, n.26, 135 - 147. Guimarães, M. (2007). A formação de educadores ambientais. Campinas : Papirus, 3e édition.

Gómez, J. G. et Bernat, F. J. M. (2010). Cómo y que enseñar de la biodiversidad en la alfabetización científica. Enseñanza de las Ciencias, vol. 28, nº 2, 175-184.

Henning, C. C., Garré B. H. et Henning, P. C. (2010). Discursos da educação ambiental na mídia : uma estratégia de controle social em operação. Rev. Eletrônica Mestr. Educ. Ambient. vol. 25, 243-252.

Kawasaki, C. S. et Oliveira, L. B. (2003). Biodiversidade e educação : as concepções de biodiversidade dos formadores de professores de biologia. Dans IV Encontro Nacional de Pesquisa em Educação em Ciências. Annales... Bauru, 2003.

Klosterman, M. L., Sadler, T. D. et Brown, J. (2012). Science Teachers' Use of Mass Media to Address Socio-Scientific and Sustainability Issues. Research in Science Education, vol. 42, $\mathrm{n}^{\circ} 1,51-74$. Marandino, M., Selles, S. et Ferreira, M. (2009). Ensino de Biologia : histórias e práticas em diferentes contextos. São Paulo : Cortez.

Reigota, M. A.(1999). A floresta e a escola : por uma educação ambiental pós-moderna. São Paulo : Cortez.

Roberto, E. C. de O. (2019). Relacionando os conhecimentos de uma mídia audiovisual do ensino médio sobre o tema biodiversidade. Mémoire de maîtrise. Université de São Paulo.

Santos, L. L. (2006). o enquadramento midiático da sustentabilidade e o papel da mídia na assimilação social do consumo sustentável. Dans XXIX Congresso Brasileiro de Ciências da comunicação, 2006, Brasília. Annales. Brasília : Intercom, 2006.

Sauvé, L (2017). Introduction. Dans : Sauvé, L., Orellana, I., Villemagne, C. et Bader, B (Dir). Éducation, Environnement, Écocitoyenneté - Repères contemporains. Presses de l'Université du Québec. Sauvé, L. (2006) Complexité et diversité du champ de l'éducation relative à l'environnement. Chemin de traverse - Revue transdisciplinaire en éducation à l'environnement 3, Solstice d'été 2006, p. 51-62.

Sauvé, L. et Orellana, I. (2008). Conjuguer rigueur, équité, créativité et amour : l'exigence de la criticité en éducation relative à l'environnement. Éducation relative à l'environnement, vol. 7, 7-20.

Silva, R. L. F (2010). Leitura de imagens da mídia e educação ambiental : contribuições para a formação de professores. Educação em Revista, vol. 26, n 2, 277-298.

Silva, R. L. F. (2007). o meio ambiente por trás da tela : estudo das concepções de educação ambiental dos filmes da TV Escola. Thèse de doctorat inédite. Université de São Paulo. 
Silva, R.L.F, Abreu, R. C.S.M. (2015). Teaching mediation of media in classes of Sciences. Dans : ESERA 2015, Helsinki. Science Education Research : Engaging Learners for a Sustainable future.

Thinen, A. C. et Silva, R. L. F. (2014). O conceito de biodiversidade retratada em uma mostra audiovisual ambiental. Revista de Ensino de Biologia - Sbenbio, $\mathrm{n}^{\circ}$ 7, 788-799.

Torales, M. A. (2013). Educação ambiental na formação de professores : compromisso políticoideológico, social e pedagógico. Ambientalmente sustentable, Oleiros, vol. 1, 31-41.

UNESCO. (2012). Éducation aux médias et à l'information : programme de formation pour les enseignants. Paris : UNESCO.

Van Der Maren, J. M. (1996). Méthodes de recherche pour l'éducation. Montréal : Les presses de l'Université de Montréal, $2^{\mathrm{e}}$ édition.

Xavier, M. E. R. et Kerr, A. S. (2004). A análise do efeito estufa em textos para-didáticos e periódicos jornalísticos. Cad. Bras. Ensino de Física. vol. 21, nº 3, 325-349.

Zacarias, R. (2009). "Sociedades de consumo" : ideologia do consumo e as iniquidades socioambientais dos atuais padrões de produção. Dans LOUREIRO, C. F. B., Layrargues, P. P., De Castro, R. S. Repensar a educação ambiental : um olhar crítico. São Paulo : Cortez.

\section{NOTES}

1. Il s'agit du premier épisode d'une série de douze reportages sur la biodiversité, présenté en 2004 par une importante chaîne de télévision brésilienne. Disponible à l'adresse suivante : https://www.youtube.com/watch?v=7mWZXY6_tlc\&t=14s (consulté le 18 juin 2020)

\section{Processus CAAE 69250517.5.0000.5464}

3. https:// www.qsrinternational.com/nvivo-qualitative-data-analysis-software/home (consulté le 18 juin 2020)

\section{RÉSUMÉS}

Cet article présente l'analyse d'une pratique de lecture critique d'un reportage télévisé qui a été développée au cours d'un atelier de formation continue des enseignant.e.s du secondaire de l'État de São Paulo au Brésil. Nous avons caractérisé les discussions entre les participant.e.s après la projection du reportage, en particulier à la lumière du courant critique de l'éducation relative à l'environnement. Au cours de leurs échanges, les enseignant.e.s ont signalé la non-neutralité de cette production médiatique et en plus d'examiner les thèmes présentés dans le reportage, les participant.e.s ont abordé de sujets n'ayant pas été traités dans le documentaire, tels que la situation politique et la crise du système économique. Nous soulignons le potentiel d'une telle activité pour promouvoir une formation continue s'inspirant des fondements d'une éducation libertaire, engagée et consciente. 
This research analyzes a practice of critical reading of a television report, which was developed during a workshop for in-service training of secondary teachers in the State of São Paulo, Brazil. We characterize the discussions developed by the teachers after the presentation of the documentary, in light of the field of critical environmental education. It was possible to realize that teachers were aware of media non-neutrality and that, in addition to criticizing the themes presented in the report, the teachers used a long period of discussion to share subjects that were not present, such as politics and the economic system crisis. We underline the potential of such an activity to promote an in-service teacher education based on the theoretical foundations of a libertarian, engaged and conscious education.

\section{INDEX}

Keywords : environmental education, media literacy, teacher training, critical reading, biodiversity, television.

Mots-clés : éducation relative à l'environnement, éducation aux médias, formation continue des enseignants, lecture critique, biodiversité, télévision.

\section{AUTEURS}

\section{MARIANA TAMBELLINI FAUSTINO}

Mariana Tambellini Faustino est diplômée en science et technologie (2011) et en biologie (2012), et détient une maîtrise en enseignement, histoire et philosophie des sciences et des mathématiques (2014) et un doctorat en enseignement des sciences (2019) à l'Université de São Paulo. Elle participe au GPEAFE et est actuellement enseignante de biologie au secondaire. Ses travaux s'intéressent notamment à l'ERE, à l'EMI et à la formation continue des enseignant.e.s.

\section{ROSANA LOURO FERREIRA SILVA}

Rosana Louro Ferreira Silva est diplômée en biologie, et détient une maîtrise en écologie (2000) et un doctorat en éducation - enseignement des sciences (2007). Elle est actuellement professeure en enseignement de la biologie au département de zoologie de l'Institut des Biosciences de l'Université de São Paulo. Elle est coordinatrice du GPEAFE et se spécialise dans les domaines de l'ERE, de l'enseignement de la biologie et de la formation des enseignant.e.s 